\title{
Balloon-Occluded Transarterial \\ Chemoembolization: In Which Size Range Does It Perform Best? A Comparison of Its Efficacy versus Conventional Transarterial Chemoembolization, Using Propensity Score Matching
}

\author{
Rita Golfieri ${ }^{a}$ Mario Bezzi ${ }^{b} \quad$ Gontran Verset $^{c} \quad$ Fabio Fucilli $^{d} \quad$ Cristina Mosconi $^{a}$ \\ Alberta Cappellia ${ }^{a}$ Alexandro Paccapelo ${ }^{a}$ Pierleone Lucatellib \\ Nicolas Magand ${ }^{e}$ Agnes Rode ${ }^{e}$ Thierry De Baere ${ }^{f}$ \\ aDepartment of Radiology, IRCCS Azienda Ospedaliero-Universitaria di Bologna, Bologna, Italy; ${ }^{b}$ Vascular and \\ Interventional Radiology Unit, Department of Diagnostic Service, Sapienza University of Rome, Rome, Italy; \\ 'Department of Gastroenterology, Hepatopancreatology and Digestive Oncology, Erasme Hospital, Université Libre \\ de Bruxelles (ULB), Brussels, Belgium; ${ }^{d}$ Radiology Unit, "S. De Bellis" National Institute of Gastroenterology Research

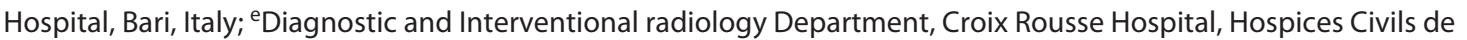 \\ Lyon, Lyon, France; 'Department of Interventional Radiology, Gustave Roussy Cancer Center, Villejuif, France
}

\section{Keywords}

Hepatocellular carcinoma - Balloon-occluded transcatheter arterial chemoembolization · Transcatheter arterial chemoembolization

\begin{abstract}
Introduction: The aim of this multicenter comparison of balloon-occluded transarterial chemoembolization (B-TACE) versus conventional TACE (CTACE) in treating hepatocellular carcinoma (HCC) was to assess in which size range the 2 techniques offered higher complete response (CR) and objective response (OR) rates in a single session, and to evaluate the possibility of using B-TACE to reduce the need for re-treatment. Methods: 325 patients were retrospectively evaluated: 91 patients in the B-TACE group (22 with cTACE [B-CTACE] and 69 with drug-eluting microsphere TACE [B-DEM-TACE]) and 234 in the CTACE group. The results were compared according to tumor size: (A) $<30 \mathrm{~mm}$, (B) 30-50 mm, and (C)
\end{abstract}

$>50 \mathrm{~mm}$; OR and $\mathrm{CR}$ rates after the first session and the number of TACE re-interventions within a 6-month period were also evaluated using propensity score matching (PSM). $\boldsymbol{R} \boldsymbol{e}$ sults: The best target ORs were very high (93.2\%) and similar between the 2 treatments both before $(94.4 \%$ for CTACE and 90.1\% for B-TACE) and after PSM (94.5\% for CTACE and $90.1 \%$; $p=0.405)$, with slightly better results for the CTACE cohort probably due to better CTACE effectiveness in smaller lesions. In lesions $<30 \mathrm{~mm}, \mathrm{cTACE}$ obtained a slightly higher CR rate than B-TACE (61.9 vs. $56.3 \%, p=0.680)$, whereas in intermediate-sized HCCs (30-50 mm), B-TACE showed a significant superiority in achieving a $C R$ (72.3 vs. $54.1 \%$, respectively; $p=0.047$ ). In larger lesions (>50 mm), CTACE and BTACE performed equally, with a poor CR rate (22.6 vs. $23.1 \%$, respectively; $p=1.000$ ). These results were additionally confirmed using PSM. The patients treated with B-TACE had a significantly lower re-treatment rate than the CTACE cohort (12.1 vs. $26.9 \%$, respectively; $p=0.005$ ). B-cTACE and B-DEMTACE demonstrated similar ORs, with a slightly better $C R$ rate karger@karger.com www.karger.com/lic

Karger $\frac{1}{\%}$

BOPEN ACCESS
(C) 2021 The Author(s)

Published by S. Karger AG, Basel

This is an Open Access article licensed under the Creative Commons Attribution-NonCommercial-4.0 International License (CC BY-NC) (http://www.karger.com/Services/OpenAccessLicense), applicable to the online version of the article only. Usage and distribution for commercial purposes requires written permission.
Correspondence to:

Rita Golfieri, rita.golfieri@unibo.it 
for B-CTACE (68.2 vs. $56.5 \%$, respectively; $p=0.456$ ). Conclusion: In HCCs of 30-50 mm, B-TACE should be preferred to CTACE, whereas in smaller nodules $(<30 \mathrm{~mm})$, CTACE can suffice in achieving a good $C R$ rate. The statistically significant lower re-treatment rate of the B-TACE cohort after a single procedure reduced the risk of complications due to multiple TACE, which could worsen the patient prognosis.

(c) 2021 The Author(s)

Published by S. Karger AG, Basel

\section{Introduction}

Transarterial chemoembolization (TACE) is the only guideline-recommended global standard of care for intermediate-stage (stage B) hepatocellular carcinoma (HCC) according to the Barcelona Clinic Liver Cancer (BCLC) staging system; however, it is also used in the early stage when ablation and resection are not indicated [14]. In fact, it has been demonstrated that TACE is the most common first treatment modality used in HCC patients, regardless of tumor stage. The BRIDGE study, an international, large-scale longitudinal cohort study, which included 18,031 patients in 14 countries, with the aim of understanding the real-life management of HCC patients, found that TACE was the most frequent treatment in North America, Europe, China, and South Korea and was used to treat nearly $50 \%$ of patients from stage 0 to stage D HCC in daily practice [5]. TACE was also the most common second treatment for HCC after percutaneous ethanol injection/radiofrequency ablation in all regions with the exception of North America where liver transplant was more common. Based on the recent European Association for the Study of the Liver (EASL) guidelines [1], either conventional TACE (cTACE) or drugeluting microsphere TACE (DEM-TACE) can be utilized, with the choice left to the physician.

A systematic review involving 101 articles from 1980 to 2013 [6] reported an objective response (OR: sum of complete response (CR) and partial response needing retreatment) rate of only $52.5 \%$. In the more recent series, the OR rate increased to nearly $100 \%$ [7-9] due to technical refinements, such as the introduction of microcatheters and to the advancement of technologies, such as cone beam computed tomography (CT) with sophisticated software for guidance of the procedures, allowing a more selective approach to target lesions. It is well known that superselective TACE, recently renamed "curative TACE" [10], aims at achieving a CR and has significantly improved overall survival (OS) compared to non-superselective TACE (“non-curative TACE”) [11-14]. In a previ- ous series with histologic confirmation on explanted livers, the tumor necrosis rate was $75.1 \%$ for selective/ superselective cTACE and 52.8\% for nonselective cTACE $(p=0.002)$, with a complete necrosis rate of 53.8 versus $29.8 \%(p=0.013)$, respectively, suggesting that tumor necrosis was more frequently achieved over a significantly wider range of lesions with selective/superselective cTACE [15].

Patients displaying a CR to initial TACE have significantly longer OS, suggesting the importance of achieving a CR in the initial TACE procedure [16]. Regrettably, TACE does not achieve a radiological CR in a satisfactory percentage of patients after a single session, and TACE repetition is frequently necessary. Unfortunately, the response rate decreases with additional TACE sessions as compared with the response to the initial TACE session $[17,18]$. In addition, repeated TACE can increase morbidity and mortality by means of TACE-induced risks, such as deterioration of the liver function. It has been reported that a non-CR after initial TACE is independently associated with large and multiple tumors $[10,16]$. The size dependence of the achievement of a CR after TACE has previously been proven in the medical literature regarding both radiology and pathology. The CR rate at 1-6 months after cTACE/DEM-TACE reported in previous randomized control trials ranged from 45 to $68 \%$ [8], with the highest numbers obtained in smaller lesions $(<2 \mathrm{~cm})$, decreasing to $64 \%$ in nodules ranging from 2.1 to $5 \mathrm{~cm}$, and decreasing additionally to very low rates (25\%) in lesions $>5 \mathrm{~cm}$ [17].

To increase the rate of a CR, especially in large tumors, combined treatments have been proposed. In HCCs ranging from 3 to $5 \mathrm{~cm}$, a combination of intra-arterial therapy and ablation seems to provide benefits regarding OS and recurrence-free survival, whereas the combination of TACE and antiangiogenic drugs in 4 trials failed to demonstrate any clinical benefit [19].

Another effort to increase the rate of a CR in TACE was the introduction of balloon-occluded TACE (B-TACE) in 2009 [20, 21]; the B-TACE procedure is performed using a balloon microcatheter inflated within the tumor-feeding arteries during selective/superselective TACE. The theoretical superiority of B-TACE is due to the hemodynamic changes caused by the balloon inflation. In B-TACE, the blocking effect of the proximal arteries decreases the balloon-occluded arterial stump pressure (BOASP). The drug-embolic mixture (Lipiodol ${ }^{\circledR}$-based or beads) can subsequently be forcefully introduced into the tumor vessels and is more intensively administered, also filling the arterioportal micro-anastomoses at the periphery of the 
Table 1. B-TACE patients coming from the different European centers

\begin{tabular}{lll}
\hline Hospital & $\begin{array}{l}\text { Patients treated, } \\
n(\%)\end{array}$ & $\begin{array}{l}\text { Patients followed up, } \\
n(\%)\end{array}$ \\
\hline IRCCS Azienda Ospedaliero-Universitaria di Bologna, ${ }^{*}$ Italy & $10(10.4)$ & $10(11.0)$ \\
Gustave Roussy Cancer Center, France & $10(10.4)$ & $9(9.9)$ \\
Sapienza University of Rome, Italy & $28(29.2)$ & $28(30.8)$ \\
Erasme Hospital Brussels, Belgium & $26(27.1)$ & $26(28.6)$ \\
IRCCS De Bellis, Castellana Grotte, Bari, Italy & $20(20.8)$ & $18(19.8)$ \\
Hôpital Croix Rousse Lyon, France & $2(2.1)$ & $0(0.0)$ \\
Overall & 96 & 91 \\
\hline
\end{tabular}

B-TACE, balloon-occluded transarterial chemoembolization. ${ }^{*}$ Coordinating sites.

tumor [22]. This has been reported in cases in which the BOASP is $64 \mathrm{~mm} \mathrm{Hg}$ or less and in the absence of large collateral arteries [21]. This allows for an enhanced therapeutic effect as compared with cTACE $[21,23]$ and for a better modified Response Evaluation Criteria in Solid Tumors (mRECIST) response with higher rates of portal vein visualization, indicating the enlargement of the area treated to include the drainage area (the site of microsatellites) [22]. The present multicenter retrospective study is the first large European comparative study of B-TACE versus CTACE, which has the aim of investigating the size ranges in which CTACE and B-TACE could offer more benefits in terms of CR rates after the first session, and exploring a possible reduction in the need for re-treatment (according to the on-demand treatment strategy).

\section{Materials and Methods}

This retrospective study complied with the principles of the Declaration of Helsinki and subsequent amendments and was carried out at tertiary liver care centers. Written informed consent for each TACE procedure was obtained from all patients. All personal data were blinded and anonymized in the general database. Institutional Review Board approval was not required as patients were treated with approved diagnostic and therapeutic procedures according to generally accepted standards of care and preliminarily discussed in multidisciplinary tumor boards of each center.

\section{Study Population}

The B-TACE study population included 91 patients ( 179 nodules) of 96 treated ( 5 were lost to follow-up), coming from 6 centers (Table 1), enrolled consecutively during the current clinical practice between January 2015 and December 2019, affected by intermediate-stage HCC (or early stage if not amenable to curative treatments), who had undergone either Lipiodol ${ }^{\circledR}$-based cTACE or DEM-TACE. The control group included 234 patients $(n=445$ nodules), taken from a historical institutional database and having similar characteristics (demographic and disease), who underwent selective/superselective cTACE using a conventional microcathe- ter (Table 2). To evaluate the effectiveness of the B-TACE and the cTACE treatments according to tumor size, the patients were stratified into 3 groups: (A) <30 mm; (B) 30-50 mm; and (C) $>50 \mathrm{~mm}$. The responses after $\mathrm{B}$-TACE, according to tumor size and the number of required re-treatments, were then compared with those obtained in the control group.

The inclusion criteria were a Child-Pugh score up to B8, a BCLC stage up to $B$, and not being eligible for curative treatment (surgical resection or percutaneous ablative treatment). Patients presenting with a Child-Pugh class $>\mathrm{B} 8$, portal vein thrombosis (defined as the complete or partial obstruction of blood flow in the main or segmental portal branches due to a chronic, acute, or neoplastic thrombus in the vasal lumen), extrahepatic metastasis, high-flow arterioportal or arteriovenous shunts, previous systemic treatment, platelet count $<50,000$, and bilirubin level $>3 \mathrm{mg} / \mathrm{dL}$ were considered to be unsuitable for the procedure. All treated lesions had previously been untreated.

\section{TACE and B-TACE Technical Procedures}

CTACE was carried out by selective catheterization of the hepatic arteries feeding the lesions; in the majority of patients, superselective or selective TACE was carried out using a highly flexible coaxial microcatheter (2.7-2.8 Fr Progreat ${ }^{\mathrm{TM}}$ Terumo or Renega$\mathrm{de}^{\mathrm{TM}}$ HI-FLO; Boston Scientific) passed through a 4 Fr catheter previously placed in the hepatic artery. For selective TACE, the tip of the microcatheter was placed into the hepatic arterial branch afferent to the segment in which the tumor was located. In superselective TACE, the tip of the catheter was additionally advanced into the subsegmental branches feeding the nodule. After the microcatheter placement, a mixture of epirubicin (Farmorubicina ${ }^{\circledR}$; Pfizer, Latina, Italy) and iodized oil (Lipiodol ${ }^{\circledR}$; Guerbet, Milan, Italy) was injected under fluoroscopic control, followed by embolization using Spongel (Gelitaspongel ${ }^{\circledR}$ ) particles until complete blockage of the tumor-feeding vessels was achieved. The endpoint of the Lipiodol ${ }^{\circledR}$-epirubicin infusion was the beginning of portal branch depiction.

The emulsion of epirubicin and Lipiodol ${ }^{\circledR}$ was mixed without the interposition of contrast medium ("iodized oil suspension"), as previously recommended [24], in all cases of the cTACE arm and in 10/22 patients of the B-cTACE arm, whereas 12/22 of BcTACE arm were treated with a water-in-oil emulsion (droplets of the internal phase containing drug in aqueous solution and continuous external phase of oily Lipiodol ${ }^{\circledR}$ ), in line with previous 


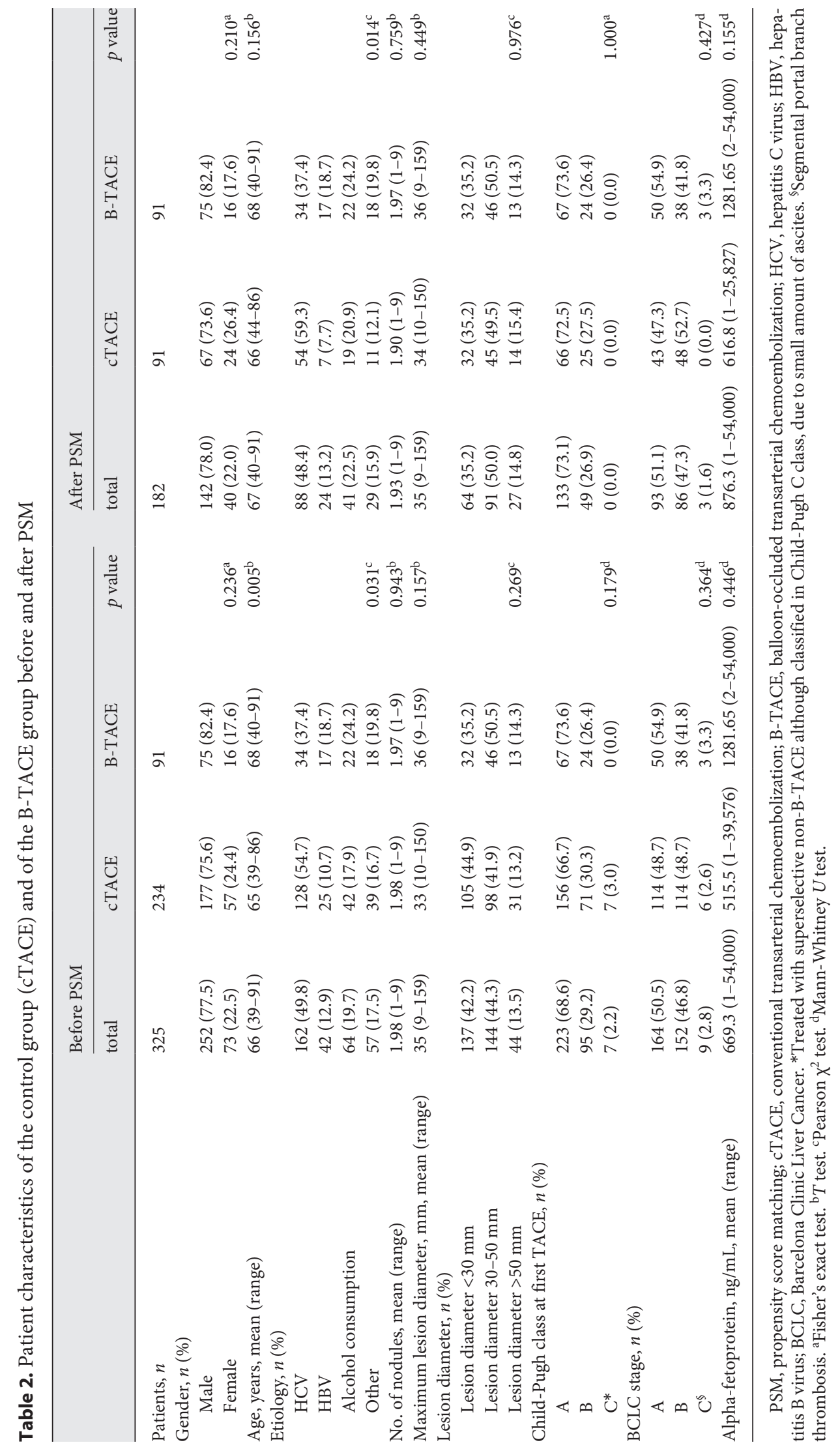


Table 3. Baseline characteristics and comparison of responses between the 2 types of B-TACE treatment (Lipiodol-based or with DEM)

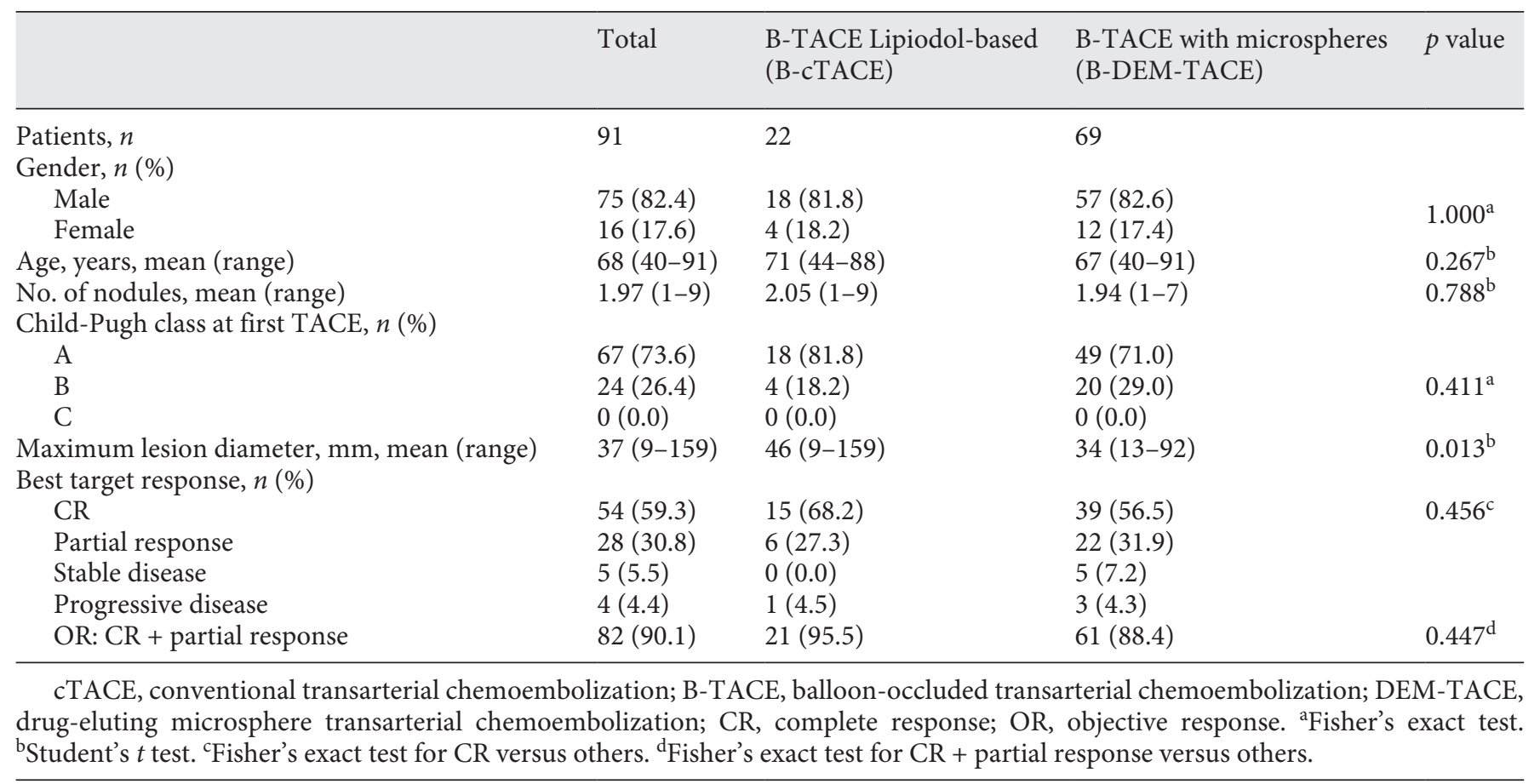

standardization protocol [25]. The gelatin sponge particles had been hand-cut, obtaining particles measuring approximately $1-1.5$ $\mathrm{mm}$, and had been mixed with a few milliliters of contrast media and were then forcefully mixed using a pumping method between 2 syringes, until crushed into approximately $0.2-0.5 \mathrm{~mm}$ particles. The mean chemotherapeutic agent dose administered per treatment was approximately $40 \mathrm{mg}$ of epirubicin (range, 20-75 mg), and the mean Lipiodol ${ }^{\circledR}$ dose administered was approximately 8 $\mathrm{mL}$ (range, 4-15 mL).

The B-TACE procedure was performed using a balloon microcatheter (Occlusafe ${ }^{\circledR}$; Terumo Europe NV, Leuven, Belgium), that is, a 2.8 Fr microcatheter with an occlusion balloon on the tip. The micro-balloon is made of compliant polyurethane and is $10 \mathrm{~mm}$ in length. The diameter ranges from 1 to $4 \mathrm{~mm}$, according to the volume injected. The micro-balloon catheter was inserted within a standard $4 \mathrm{Fr}$ angiographic catheter using a coaxial technique. The balloon microcatheter works on a 0.014 " platform; in this series, all the procedures were performed using a hydrophilic guidewire. The micro-balloon inflation was carried out with a solution of 1:4 of contrast media/saline. The balloon was inflated to occlude the flow and to obtain a drop in the BOASP. The BOASP was measured before and after inflation of the balloon. The embolization was then performed according to routine clinical practice as described above.

Assessment of Tumor Radiological Response and Follow-Up

Patients underwent imaging assessment (quadriphasic CT or dynamic magnetic resonance imaging) 1 month after TACE in order to evaluate the radiological response according to the mRECIST criteria [26], measuring the best target response observed in the follow-up period of 6 months (at 1, 3, and 6 months) in accor- dance with the current guidelines. The OR rate was considered to be the percentage of patients with a CR or a partial response of the target lesion(s) (maximum 2 lesions) obtained during the followup period.

A follow-up of 6 months was also considered for the re-treatment needed for residual tumor or recurrences on target pretreated lesion(s). When viable hepatic nodules were detected on followup CT/magnetic resonance imaging, on-demand TACE was performed in the majority of cases as long as the liver function was Child-Pugh A/B and a portal venous thrombus was not seen in the lobar branch or main trunk.

\section{Study Safety Outcomes}

The study recorded the incidence of biological and clinical adverse events (AEs) according to the CIRSE classification system and Common Terminology Criteria for Adverse Events (CTCAE) version $5.0[27,28]$ occurring within 30 days post-procedure, based on information collected regarding the event. Post-embolization syndrome (PES) was defined as the onset of fever, nausea/ vomiting, and pain and was clinically evaluated during the patient's hospital stay. The radiological safety evaluation carried out at the 1-month follow-up included the detection of liver bile duct injuries, such as segmental dilation or biloma formation, liver infarction in the non-tumoral parenchyma, and the appearance of indirect imaging features of vascular damage.

\section{Statistical Analysis}

Data are presented as mean and range for the continuous variables, and as absolute numbers and percentages for the categorical variables. A propensity score matching (PSM) procedure was subsequently performed in a 1:1 ratio for age, gender, number of nod- 
Table 4. Best target response according to tumor dimensions before and after PSM

\begin{tabular}{|c|c|c|c|c|c|c|c|c|}
\hline & \multicolumn{4}{|c|}{ Before PSM, $n(\%)$} & \multicolumn{4}{|c|}{ After PSM, $n(\%)$} \\
\hline & total & cTACE & B-TACE & $p$ value & total & cTACE & B-TACE & $p$ value \\
\hline Patients, $n$ & 325 & 234 & 91 & & 182 & 91 & 91 & \\
\hline $\mathrm{CR}$ & $179(55.1)$ & $125(53.4)$ & $54(59.3)$ & $0.385^{\mathrm{a}}$ & $99(54.4)$ & $45(49.5)$ & $54(59.3)$ & $0.234^{\mathrm{a}}$ \\
\hline Partial response & $124(38.2)$ & $96(41.0)$ & $28(30.8)$ & & $69(37.9)$ & $41(45.1)$ & $28(30.8)$ & \\
\hline Stable disease & $7(2.2)$ & $2(0.9)$ & $5(5.5)$ & & $5(2.7)$ & $0(0.0)$ & $5(5.5)$ & \\
\hline Progressive disease & $15(4.6)$ & $11(4.7)$ & $4(4.4)$ & & $9(4.9)$ & $5(5.7)$ & $4(4.4)$ & \\
\hline OR: CR + partial response & $303(93.2)$ & $221(94.4)$ & $82(90.1)$ & $0.217^{\mathrm{b}}$ & $168(92.3)$ & $86(94.5)$ & $82(90.1)$ & $0.405^{\mathrm{b}}$ \\
\hline Lesion diameter $<30 \mathrm{~mm}$ & 137 & 105 & 32 & & 64 & 32 & 32 & \\
\hline CR & $83(60.6)$ & 65 (61.9) & $18(56.3)$ & $0.680^{\mathrm{a}}$ & $38(59.4)$ & $20(62.5)$ & $18(56.3)$ & $0.799^{\mathrm{a}}$ \\
\hline Partial response & $46(33.6)$ & $37(35.2)$ & $9(28.1)$ & & $20(31.3)$ & $11(34.4)$ & $9(28.1)$ & \\
\hline Stable disease & $5(3.6)$ & $2(1.9)$ & $3(9.4)$ & & $3(4.7)$ & $0(0.0)$ & $3(9.4)$ & \\
\hline Progressive disease & $3(2.2)$ & $1(1.0)$ & $2(6.3)$ & & $3(4.7)$ & $1(3.1)$ & $2(6.3)$ & \\
\hline OR: CR + partial response & $129(94.2)$ & $102(97.1)$ & $27(84.4)$ & $0.017^{\mathrm{b}}$ & $129(94.2)$ & $31(96.9)$ & $27(84.4)$ & $0.196^{\mathrm{b}}$ \\
\hline Lesion diameter $30-50 \mathrm{~mm}$ & 144 & 98 & 46 & & 91 & 45 & 46 & \\
\hline CR & $86(59.7)$ & $53(54.1)$ & $33(71.7)$ & $0.047^{\mathrm{a}}$ & $55(60.4)$ & $22(48.9)$ & $33(71.7)$ & $0.033^{\mathrm{a}}$ \\
\hline Partial response & $49(34.0)$ & $39(39.8)$ & $10(21.7)$ & & $32(35.2)$ & $22(48.9)$ & $10(21.7)$ & \\
\hline Stable disease & $2(1.4)$ & $0(0.0)$ & $2(4.3)$ & & $2(2.2)$ & $0(0.0)$ & $2(4.3)$ & \\
\hline Progressive disease & $7(4.9)$ & $6(6.1)$ & $1(2.1)$ & & $2(4.9)$ & $1(2.2)$ & $1(2.1)$ & \\
\hline OR: $\mathrm{CR}$ + partial response & $135(93.8)$ & $92(93.9)$ & $43(93.5)$ & $1.000^{\mathrm{b}}$ & $87(93.8)$ & $44(97.8)$ & $43(93.5)$ & $0.617^{\mathrm{b}}$ \\
\hline Lesion diameter $>50 \mathrm{~mm}$ & 44 & 31 & 13 & & 27 & 14 & 13 & \\
\hline $\mathrm{CR}$ & $10(22.7)$ & $7(22.6)$ & $3(23.1)$ & $1.000^{\mathrm{a}}$ & $6(22.2)$ & $3(21.4)$ & $3(23.1)$ & $1.000^{\mathrm{a}}$ \\
\hline Partial response & $29(65.9)$ & $20(64.5)$ & $9(69.2)$ & & $17(63.0)$ & $8(57.1)$ & $9(69.2)$ & \\
\hline Stable disease & $0(0.0)$ & $0(0.0)$ & $0(0.0)$ & & $0(0.0)$ & $0(0.0)$ & $0(0.0)$ & \\
\hline Progressive disease & $5(11.4)$ & $4(12.9)$ & $1(7.7)$ & & $4(14.8)$ & $3(21.4)$ & $1(7.7)$ & \\
\hline OR: CR + partial response & $39(88.6)$ & $27(87.1)$ & $12(92.3)$ & $1.000^{\mathrm{b}}$ & $23(85.2)$ & $11(78.6)$ & $12(92.3)$ & $0.596^{\mathrm{b}}$ \\
\hline
\end{tabular}

PSM, propensity score matching; cTACE, conventional transarterial chemoembolization; B-TACE, balloon-occluded transarterial

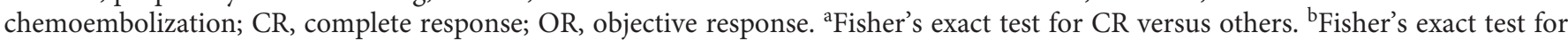
complete + partial response versus others.

ules, and Child-Pugh score, with a match tolerance of 0.2 giving priority to exact matches. Baseline patient characteristics and the best target responses according to tumor size were analyzed before and after the PSM, and AEs were evaluated in the PSM selected population. The categorical variables were analyzed using the $\chi^{2}$ test or the Fisher's exact test as appropriate; the ordinal data were compared using the Mann-Whitney $U$ test. The continuous variables were compared using the Student's $t$ test. All the tests were 2 -tailed, and a $p$ value of $<0.05$ was considered statistically significant. All the statistical analyses were carried out using IBM SPSS 25.0 (SPSS Inc., Armonk, NY, USA).

\section{Results}

\section{Patient Population}

The demographic distribution and the patient characteristics of the B-TACE and the cTACE patient cohorts before and after PSM are shown in Table 2. In the BTACE group, 22 patients underwent B-cTACE and 69 were treated with B-DEM-TACE. In the CTACE treat- ments, a mean dose of $7.7 \mathrm{~mL}$ of Lipiodol ${ }^{\circledR}$ was injected (range 2-15 mL) mixed with a mean epirubicin dose of $41.1 \mathrm{mg}$ (range $17-100 \mathrm{mg}$ ). In the B-DEM-TACE patients, Life Pearls $100 \mu \mathrm{m}$ loaded with an anthracycline mean dose of $33.5 \mathrm{mg}$ and Life Pearls $200 \mu \mathrm{m}$ with an anthracycline mean dose of $23.5 \mathrm{mg}$ were injected. The treatment duration of B-TACE and cTACE procedures was similar.

\section{Treatments and Tumor Response}

The demographic characteristics and the type of treatment performed under balloon occlusion (B-cTACE or B-DEM-TACE) did not impact the response rates (Table 3), although statistically nonsignificant better responses were observed in the B-cTACE cohort (68.2 vs. $56.5 \%$, respectively; $p=0.456$ ). In all 325 patients, regardless of the treatment arm, the best target ORs (Table 4) were very high (93.2\%) and were similar between the 2 treatments (94.4\% for cTACE and $90.1 \%$ for B-TACE); after PSM, these numbers were additionally confirmed 
(94.5\% for cTACE and $90.1 \% ; p=0.405)$, with slightly better results for cTACE.

On evaluation of the results of B-TACE across tumor sizes (Table 4), in smaller lesions ( $<30 \mathrm{~mm}$ ), before PSM, cTACE and B-TACE achieved similar CRs (61.9 and $56.3 \%$, respectively; $p=0.680$ ), and after PSM, these results were confirmed (62.5\% for cTACE and $56.3 \%$ for $\mathrm{B}$-TACE; $p=0.799$ ). Conversely, in the $30-50 \mathrm{~mm}$ size range, both before and after PSM, the CR rates were significantly higher in the B-TACE arm (71.7 vs. $54.1 \%$, respectively [ $p=0.047]$, before PSM, and 71.7 vs. $48.9 \%$, respectively $[p=0.033]$, after PSM). In the larger lesions $(>50 \mathrm{~mm})$, cTACE and B-TACE performed equally, with very poor CRs in both arms (22.6\% in cTACE and $23.1 \%$ in B-TACE).

In the B-TACE patients, the BOASP with the microballoon inflated was $64.1 \pm 27.7 \mathrm{~mm} \mathrm{Hg}$ (minimum 33 $\mathrm{mm} \mathrm{Hg}$ and maximum $220 \mathrm{~mm} \mathrm{Hg}$ ), while prior to inflation, it was $120.5 \pm 36.5 \mathrm{~mm} \mathrm{Hg}$; therefore, the average pressure drop was $56.4 \pm 19.6 \mathrm{~mm} \mathrm{Hg}$. No significant differences were observed between a BOASP value $<$ or $>64$ $\mathrm{mm} \mathrm{Hg}$ as a cutoff point (the CR rate: 67.9 vs. $58.8 \%$, respectively; $p=0.749$, and the OR rate: 100.0 vs. $88.2 \%$, respectively; $p=0.137$ ).

By comparing the CTACE and the B-TACE cohorts, the patients who underwent B-TACE had a significantly lower re-treatment rate within the first 6 months as compared to the patients who underwent cTACE (12.1 vs. $26.9 \%$, respectively; $p=0.005$ ) (Table 5). The AEs were reported only in the PSM cohorts (Table 6) in order to avoid selection biases. The AEs were similar between the 2 arms, with PES in $30.8 \%$ after cTACE and in $41.8 \%$ after $\mathrm{B}$-TACE, with a nonsignificant prevalence of abdominal pain and nausea in the B-TACE patients, all of whom were medically handled. All of these complications were grade 1-2 according to CTCAE version 5 . In terms of radiological complications at 1 month, CT showed the development of 2 asymptomatic abscesses (2/91 procedures evaluated: $2.2 \%$ ) in the B-TACE arm and 1 hepatic pseudoaneurysm (1.1\%) in the B-TACE arm.

\section{Discussion}

In recent years, many attempts have been made to increase TACE efficacy in terms of CR and OR, and to reduce the adverse effects on the non-cancerous liver parenchyma by means of the extensive use of thin $(2 \mathrm{~F} / 3 \mathrm{~F})$ microcatheters $[29,30]$. These efforts have increased the OR to close to $100 \%$ and the CR up to over $60 \%[7,8]$;
Table 5. Comparison of the number of re-treatments between cTACE and B-TACE

\begin{tabular}{lllll}
\hline & Total & cTACE & B-TACE & $p$ value \\
\hline Patients, $n$ & 325 & 234 & 91 & \\
Re-treatments, $n(\%)$ & $74(22.8)$ & $63(26.9)$ & $11(12.1)$ & $0.005^{\text {a }}$ \\
\hline
\end{tabular}

cTACE, conventional transarterial chemoembolization; BTACE, balloon-occluded transarterial chemoembolization. ${ }^{\mathrm{a}}$ Fisher's exact test.

these figures were also confirmed in the present study. The best target ORs in the present series were very high (93.2\%) and were similar between the 2 treatments both before (94.4\% for cTACE; $90.1 \%$ for B-TACE) and after PSM (94.5\% for CTACE; 90.1\%; $p=0.405)$, with slightly better results for the CTACE arm, probably due to the better CTACE effectiveness in smaller lesions, having a tendency to decrease in parallel with an increase in lesion size.

A CR to the initial TACE is related to longer OS [16]; this highlights the importance of achieving a CR in the initial TACE procedure. The CR rate is directly related to a proper selection of patients, and the size and number of the tumors; a prediction of responding and refractory patients and tumors is crucial for choosing the best initial treatment to adopt. Large $(>5 \mathrm{~cm})$ and multiple $(\geq 4)$ tumors are independently associated with a non-CR after initial TACE $[10,16]$. Refractory tumors are those showing incomplete necrosis after $>2$ consecutive TACE procedures or patients showing $>2$ consecutive new HCC intrahepatic lesions [10,31]. According to the current EASL guidelines, TACE should not be repeated when substantial necrosis is not achieved after 2 rounds of treatment or when follow-up treatment fails to induce marked necrosis at sites which have progressed after an initial tumor response $[1,32]$. The rationale for this recommendation is based on the experience that the OR rate decreases with additional TACE sessions as compared with a response to the initial TACE $[17,18]$. Moreover, repeated TACE can increase morbidity and mortality by means of TACE-induced risks, such as deterioration of the liver function [10].

The introduction of B-TACE by Irie et al. in 2009 had the aim of additionally increasing the $\mathrm{CR}$ rate while reducing the local residual/recurrence rate. The B-TACE procedure is carried out using a balloon microcatheter inflated within the tumor-feeding arteries during selective TACE $[20,21]$, which, by modifying the flow, in- 
Table 6. AEs in the PSM selected population

\begin{tabular}{|c|c|c|c|c|}
\hline & $\begin{array}{l}\text { Patients population, } \\
n(\%)\end{array}$ & $\begin{array}{l}\text { cTACE, } \\
n(\%)\end{array}$ & $\begin{array}{l}\text { B-TACE, } \\
n(\%)\end{array}$ & $\begin{array}{l}p \\
\text { value }\end{array}$ \\
\hline All patients & 182 & 91 & 91 & \\
\hline \multicolumn{5}{|l|}{ Clinical } \\
\hline PES & $66(36.3)$ & $28(30.8)$ & $38(41.8)$ & $0.165^{\mathrm{a}}$ \\
\hline Fever & $15(8.2)$ & $10(11.0)$ & $5(5.5)$ & $0.281^{\mathrm{a}}$ \\
\hline Vomiting & $5(2.7)$ & $1(1.1)$ & $4(4.4)$ & $0.368^{\mathrm{a}}$ \\
\hline Nausea & $11(6.0)$ & $2(2.2)$ & $9(9.9)$ & $0.058^{\mathrm{a}}$ \\
\hline Abdominal pain & $31(17.0)$ & $11(12.1)$ & $20(22.0)$ & $0.114^{\mathrm{a}}$ \\
\hline Diarrhea & $0(0.0)$ & $0(0.0)$ & $0(0.0)$ & - \\
\hline Fatigue & $4(2.2)$ & $4(4.4)$ & $0(0.0)$ & $0.121^{\mathrm{a}}$ \\
\hline \multicolumn{5}{|l|}{ Biological } \\
\hline Increased ALT & $7(3.8)$ & $3(3.3)$ & $4(4.4)$ & $1.000^{\mathrm{a}}$ \\
\hline Liver function worsening (increase in Child-Pugh score of $\geq 2$ points) & $12(6.6)$ & $5(5.5)$ & $7(7.7)$ & $0.767^{\mathrm{a}}$ \\
\hline \multicolumn{5}{|l|}{ Radiological } \\
\hline Liver abscess & $2(1.1)$ & $0(0.0)$ & $2(2.2)$ & $0.497^{\mathrm{a}}$ \\
\hline Cholecystitis & $1(0.5)$ & $1(1.1)$ & $0(0.0)$ & $1.000^{\mathrm{a}}$ \\
\hline Hematoma & $1(0.5)$ & $1(1.1)$ & $0(0.0)$ & $1.000^{\mathrm{a}}$ \\
\hline Intrahepatic arterial pseudoaneurysm & $1(0.5)$ & $0(0.0)$ & $1(1.1)$ & $1.000^{\mathrm{a}}$ \\
\hline
\end{tabular}

AE, adverse event; PSM, propensity score matching; cTACE, conventional transarterial chemoembolization; B-TACE, balloon-occluded transarterial chemoembolization; PES, post-embolization syndrome. ${ }^{\text {a}}$ Fisher's exact test.

creases tumor coverage by the drug while preventing the backflow of embolic material proximally.

Lipiodol ${ }^{\circledR}$-based B-TACE has previously been confirmed to be safe and effective, achieving a higher drug concentration within the tumor [22]. Several retrospective reports, obtained in small Asian cohorts, compared results on patients treated with B-cTACE with a matched cohort treated with cTACE and demonstrated an improved therapeutic effect of B-TACE over CTACE $[22,23$, 33-35]. This is the first European multicentric retrospective comparison of B-TACE, using either a Lipiodol ${ }^{\circledR}$ drug mixture or beads loaded with drugs, versus cTACE; inclusion in the B-TACE arm of both B-cTACE $(n=22)$ and B-DEM-TACE $(n=69)$ treatments represents a novelty in the literature.

As expected, in the B-TACE arm, the type of treatment, B-cTACE or B-DEM-TACE, did not significantly impact the response rates. The equivalence in efficacy between cTACE and DEM-TACE has been demonstrated in previous randomized control trials and meta-analyses $[8,36-38]$; therefore, it is not surprising that also under balloon occlusion both treatments are almost equivalent and are only tumor size-dependent.

However, a slightly better CR in the B-cTACE versus B-DEM-TACE arm (68.2 vs. $56.5 \%$, respectively; $p=$ 0.456 ) has been observed, and this could have been due to the mean lesion diameter, which was significantly larger in the B-cTACE arm (mean 46 vs. $34 \mathrm{~mm} ; p=$ $0.013)$. Therefore, larger lesions $(30-50 \mathrm{~mm})$ as compared to smaller lesions $(<30 \mathrm{~mm})$ play an important role in achieving higher $\mathrm{CR}$ rates, thus additionally confirming the authors' size-based global results on BTACE.

In the B-TACE arm, the present OR results of $90.1 \%$ in the B-DEM-TACE arm were in line with the best responses in the literature and parallel to those of 3 recent small series on B-DEM-TACE. In a series of 22 patients treated with B-DEM-TACE, Lucatelli et al. [39] reported an OR rate of $90.9-76.5 \%$ at 1 and 3-6 months, and Goldman et al. [40] reported an OR rate of $93.3 \%$ after BDEM-TACE in 26 patients. The results of both these authors were slightly higher than those of Bucalau et al. [41] who reported a 1 -month OR rate of $74.3 \%$ in a prospective study of B-DEM-TACE on 24 patients. These numbers were far higher than those preliminarily reported by other authors [35, 42-44] after B-TACE using a miriplatin-Lipiodol ${ }^{\circledR}$ mixture (ORs of 63.6, 59.6, 57.1, and 56.3\%, respectively). However, the entire B-TACE population, regardless of the type of emulsion injected, had a significantly lower re-treatment rate within the first 6 months as compared to patients undergoing cTACE (12.1 vs. $26.9 \%$, respectively; $p=0.005$ ). 

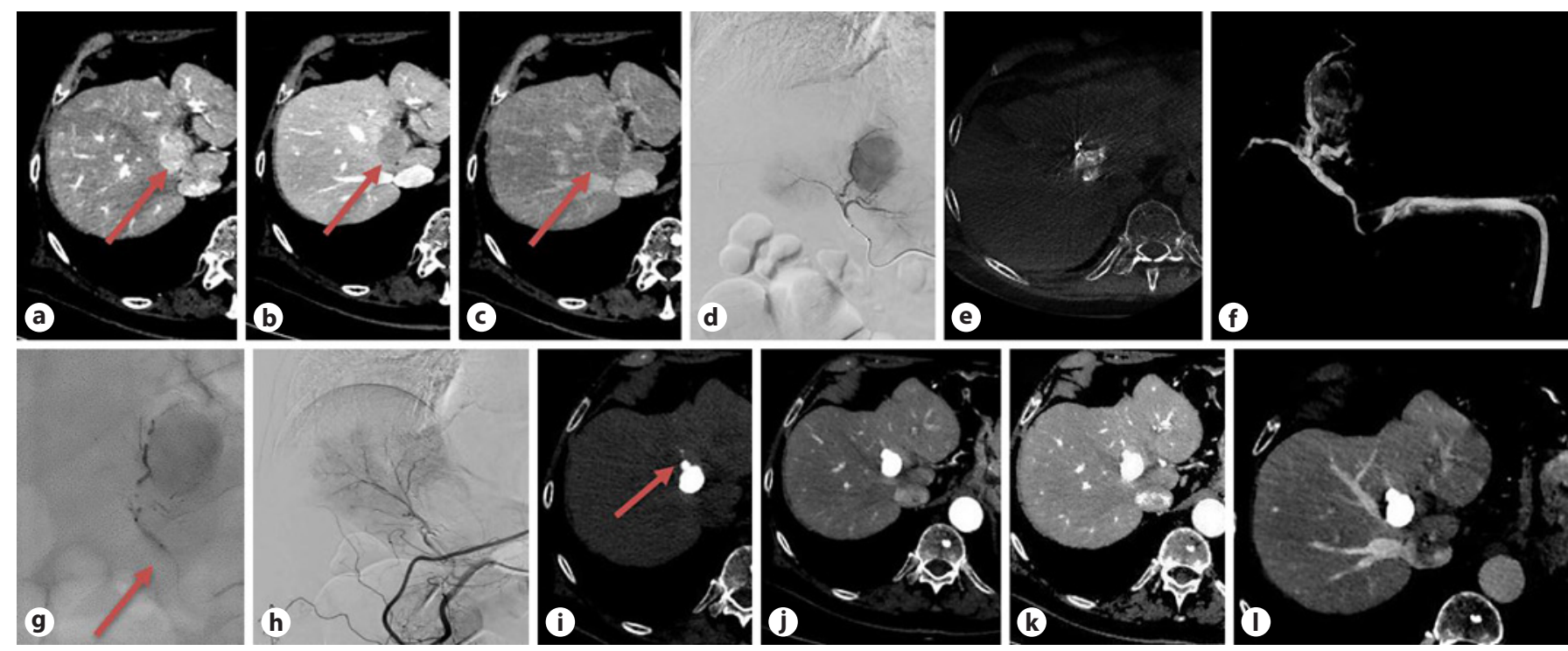

Fig. 1. Pretreatment CT: a 36-mm HCC is seen in segment IV (a, b), highly hypervascular in the arterial phase (a), rapid washout in the portal phase (b) with a corona enhancement in the late phase (c) (arrows); (d) pretreatment angiogram and cone beam CT $(\mathbf{e}, \mathbf{f})$ during balloon inflation; B-TACE treatment with Lipiodol ${ }^{\circledR}$-epirubicin injection during balloon inflation (arrow) filling the arterioportal anastomosis in the drainage area (g); final angiographic control (h); follow-up CT at 1 month (precontrast (i) and arterial (j) phase) showing a size reduction to $24 \mathrm{~mm}$, a dense distribution of Lipiodol ${ }^{\circledR}$, also including a daughter nodule in the drainage area $(\operatorname{arrow})(\mathbf{i}, \mathbf{j})$; follow-up CT at 6 months: an additional decrease in size to $22 \mathrm{~mm}$, no viable tumor (k, I). HCC, hepatocellular carcinoma; CT, computed tomography; B-TACE, balloon-occluded transarterial chemoembolization.

The size-based analysis of the best target responses in the present study demonstrated similar, or even slightly better, CR rates of cTACE before and after PSM (61.9\% before and $62.5 \%$ after PSM) when compared to B-TACE (CR 56.3\%) in smaller nodules $(<3 \mathrm{~cm})$. This could be due to the property of Lipiodol ${ }^{\circledR}$, as recently pointed out in a preclinical review of animal studies [45], to accumulate in portal venules, sinusoids, and the peribiliary plexus after arterial injection of oil-in-water and water-in-oil emulsions $[25,26]$. Oil droplets larger than sinusoids $(5-8 \mu \mathrm{m})$ blocked the sinusoids, stopping or reducing intratumoral blood flow; oil droplets smaller than sinusoids $(1-3 \mu \mathrm{m})$ circulated into the border tumor vasculature. Therefore, in smaller tumors $(<3 \mathrm{~cm})$, CTACE achieves high rates of CR since both tumor and microsatellites can be treated, as microsatellites are strictly adjacent to the tumor border. In fact, several studies have addressed the linear correlation between tumor size and the microvascular invasion of HCC cells and microsatellites. Tsai et al. [46] showed an increased incidence in microvascular invasion proportional to tumor size; $40.5 \%$ in patients with a tumor $<2 \mathrm{~cm}, 49.6 \%$ in patients with a tumor $2-4 \mathrm{~cm}$, and $58.1 \%$ in patients with a tumor $4-6 \mathrm{~cm}$ in dimension. Esnaola et al. [47] found the frequency of microvascular in- vasion to be 25,31 , and $50 \%$ for tumors $<2,2-4$, and $>4$ $\mathrm{cm}$ in their greatest dimension, respectively. The study of Sasaki et al. [48] on microsatellitosis in patients with HCCs $<5 \mathrm{~cm}$ demonstrated that tumor size and distance to the microsatellite were significantly correlated. In tumors $<2.5 \mathrm{~cm}$, the microsatellites were located within 5 $\mathrm{mm}$, and in tumors $>25 \mathrm{~mm}$, the microsatellites were located at a distance $>5 \mathrm{~mm}$. The OS rate of patients with a microsatellite distance of $>5 \mathrm{~mm}$ was lower than that of patients with a microsatellite distance $<5 \mathrm{~mm}$. In fact, it has previously been demonstrated [17] that lesions ranging 3-5 cm were the worst responding to CTACE; this could be related to a failure in treating microsatellites. Since B-TACE, due to the hemodynamic changes described, can enlarge the area treated around the tumor to include the drainage area, it was hypothesized that its superiority in achieving significantly higher CR rates than CTACE in the $30-50 \mathrm{~mm}$ size range in the present study could have been due to the potential of also treating the drainage area of tumors, site of satellite nodules even far from the primary tumor, in the same session.

The present experience demonstrated a significant superiority of B-TACE over cTACE in treating HCCs of intermediate size $(30-50 \mathrm{~mm})$, with significantly higher 
CR rates both before and after PSM $(71.7 \%$ vs. cTACE $54.1 \%$ [ $p=0.047]$ and $48.9 \%$, [ $p=0.033]$ respectively). The benefit of obtaining this result in only one session means the possibility of avoiding TACE re-treatment or combined treatments.

The present study proved that in patients undergoing B-TACE, a benefit in terms of re-treatment rates when compared to cTACE with standard microcatheters (12.1 vs. $26.9 \%$, respectively; $p=0.005$ ) was obtained due to the lower rate of residual tumor, regardless of the size of the lesion. In several previous papers, CTACE has been reported as achieving radiological CR and complete necrosis at histology of between 53.8 and $68 \%$, respectively, in small tumors $(<20-30 \mathrm{~mm})[15,17,49,50]$; in the present study, cTACE achieved very high CRs in the $<30 \mathrm{~mm}$ size range $(61.9 \%$ before and $62.5 \%$ after PSM). Therefore, cTACE was confirmed to be an effective treatment for the size range $<30 \mathrm{~mm}$. Lipiodol ${ }^{\circledR}$ is still consistently used in TACE procedures in both Eastern and Western countries, owing to its following 2 properties. First, Lipiodol ${ }^{\circledR}$ obstructs the thinner feeding arteries and the accompanying portal veins through the peribiliary arterial plexus, in which stasis is better maintained after embolization. Second, a dose of Lipiodol ${ }^{\circledR}$ drains from the tumor via the sinusoids to the peritumoral portal vessels and deposits into the surrounding parenchyma, which usually hosts satellite nodules (shown in Fig. 1), and areas of extracapsular invasion, which are also fed by the portal venous flow $[29,51,52]$. In cases in which there was marked visualization of the portal vein in the area surrounding the embolized area after the injection of Lipiodol ${ }^{\circledR}$, massive peritumoral necrosis was more frequently observed [17, 53]. Infarction of the tumor and of the surrounding parenchyma should occur, a process called "medical segmentectomy" [54], which also results in blockage of the development of new collaterals around the tumor. Therefore, complete tumor necrosis cannot be achieved when only the arterial flow is embolized [55].

The recurrence rate reported in the literature increases with tumor size, that is, 20,27 , and $67 \%$ in tumors $<2$, $2.1-5$, and $>5 \mathrm{~cm}$, respectively [17]. Incomplete TACE induces hypoxic and chemotherapeutic stress on HCC cells, and the surviving hypoxic tumors frequently change to sarcomatous or mixed hepatocholangiocellular phenotypes; in addition, it stimulates the vascular endothelial growth factor, which additionally promotes tumor progression [56-59]. It is thought that these factors constitute the basis for the development of TACE refractoriness. In patients with local tumor progression after an initial TACE, the frequency of intrahepatic distant recur- rence is significantly higher as compared with those patients with no local tumor progression [60]. A previous paper [9] has demonstrated that after CTACE of single nodules, the most statistically significant predictor of a CR was a tumor diameter $>3 \mathrm{~cm}$ (achieving a CR in 65 vs. $30 \%$ in nodules $<3 \mathrm{~cm}$ ) or that equal to or $>5 \mathrm{~cm}$, obtaining a CR 92 vs. $3 \%$ of patients, respectively.

The safety of B-TACE was also acceptable, with no intraprocedural complications in $100 \%$ of patients. Severe AEs were rare and were analogous between the B-TACE and the cTACE arms, with a prevalence of PES such as nausea (9.9\%) and abdominal pain $(22.2 \%)$ of the BTACE patients (Table 6), possibly related to the higher drug infusion and absorption in the tumor, as has already been demonstrated [22]. However, each aspect of the PES had a lower incidence compared with the literature regarding B-TACE with miriplatin $[34,61]$ and parallel the data from the 3 recent papers on B-DEM-TACE [39-41]. The first paper from Bucalau et al. [41] specifically described the safety results of B-DEM-TACE in a prospective study of 24 patients. B-DEM-TACE was shown to be safe, since they reported clinical grade $1 / 2$ toxicities $(0 \%$ $>$ grade 2 ) in $25.7 \%$ of patients, with abdominal pain being the most frequent complication (17.1\%). No 30-day mortalities or liver decompensation were observed. Another paper on B-TACE from Lucatelli et al. [39], after B-DEMTACE in a series of 22 patients, reported that laboratory test modifications were all grade 1 . AEs occurred in $4 / 24$ (17\%): pseudo-aneurysm of the feeder (grade 3), liver abscess (grade 2), and 2 asymptomatic segmentary biliary tree dilatations (grade 2). PES occurred in 8/24 (33\%). A further paper from Goldman et al. [40] in 26 patients (13 treated with B-DEM-TACE) reported the procedure as safe, with laboratory test modifications all of grade 1 . There were no major AEs and 1 minor AE; 1 patient's follow-up imaging demonstrated left portal vein thrombosis and a small liver infarct.

The present study had 3 limitations. The first was related to the retrospective analysis of the data collection, which included a wide range of lesion dimensions; this was however overcome by the PSM performed both on patients' demographics and on the subgrouping of lesions according to size. The second limitation may have derived from the inclusion of 2 different types of B-TACE procedures (B-cTACE and B-DEM-TACE): however, this can represent a novelty of the study and further confirmed the equivalence of the clinical results of both methods, as already demonstrated in non-balloon-occluded TACE. The third can be due to the different methods of emulsion of epirubicin and Lipiodol ${ }^{\circledR}$ : all cases of 
cTACE arm were performed injecting an iodized oil suspension, whereas about half of B-cTACE arm were treated with a water-in-oil emulsion. To confirm these promising retrospective matched cohort results, future multicentric randomized controlled trials are needed, focusing on specific and clinically relevant outcomes, and comparing results of B-cTACE to B-DEM-TACE in larger sample population.

\section{Conclusion}

Given the importance of selecting the proper treatment for HCC and the well-known size dependency of response to TACE, the present study demonstrated that in small lesions $(<30 \mathrm{~mm})$, cTACE can suffice since it performs very well, with similar rates of CRs when compared to B-TACE. Instead, in lesions between 30 and $50 \mathrm{~mm}$, B-TACE should be chosen since it outperformed cTACE in CR. Therefore, this could be the best lesion range to most benefit from the use of B-TACE. In lesions $>50 \mathrm{~mm}$, $\mathrm{B}-\mathrm{TACE}$ and CTACE perform equally, but with a very low $\mathrm{CR}$ rates in the range of $22-23 \%$; in this size range, a combination strategy is warranted. Moreover, regardless of the dimensional range, patients undergoing B-TACE had a benefit in terms of lower re-treatment rates when com- pared to standard cTACE, which could help in preserving liver function and in reducing rehospitalizations for retreatments.

\section{Statement of Ethics}

The study was carried out retrospectively after approval of the Institutional Ethics Committees of the hospitals involved and in compliance with the Declaration of Helsinki. Written informed consent for the procedure was obtained from all patients.

\section{Conflict of Interest Statement}

The authors have no conflicts of interest to declare.

\section{Funding Sources}

No external funding was received for this study.

\section{Author Contributions}

Conceptualization: R.G. and T.B. Data curation and methodology: A.P. Validation: M.B., G.V., F.F., P.L., N.M., and A.R. Writing - original draft: R.G., C.M., and A.C.

\section{References}

1 European Association for the Study of the Liver. EASL clinical practice guidelines: management of hepatocellular carcinoma. J Hepatol. 2018;69(1):182-236.

2 Marrero JA, Kulik LM, Sirlin CB, Zhu AX, Finn RS, Abecassis MM, et al. Diagnosis, staging, and management of hepatocellular carcinoma: 2018 practice guidance by the American association for the study of liver diseases. Hepatology. 2018;68(2):723-50.

3 Omata M, Cheng AL, Kokudo N, Kudo M, Lee JM, Jia J, et al. Asia-Pacific clinical practice guidelines on the management of hepatocellular carcinoma: a 2017 update. Hepatol Int. 2017;11(4):317-70.

4 Korean Liver Cancer Association; National Cancer Center. 2018 Korean liver cancer association-national cancer center Korea practice guidelines for the management of hepatocellular carcinoma. Gut Liver. 2019;13(3): 227-99.

5 Park JW, Chen M, Colombo M, Roberts LR, Schwartz M, Chen PJ, et al. Global patterns of hepatocellular carcinoma management from diagnosis to death: the BRIDGE Study. Liver Int. 2015;35(9):2155-66.

6 Lencioni R, de Baere T, Soulen MC, Rilling WS, Geschwind JF. Lipiodol transarterial che- moembolization for hepatocellular carcinoma: a systematic review of efficacy and safety data. Hepatology. 2016;64(1):106-16.

7 Trevisani F, Golfieri R. Lipiodol transarterial chemoembolization for hepatocellular carcinoma: where are we now? Hepatology. 2016; 64(1):23-5.

8 Golfieri R, Giampalma E, Renzulli M, Cioni $\mathrm{R}$, Bargellini I, Bartolozzi C, et al. Randomised controlled trial of doxorubicin-eluting beads vs conventional chemoembolisation for hepatocellular carcinoma. Br J Cancer. 2014; 111(2):255-64.

9 Terzi E, Piscaglia F, Forlani L, Mosconi C, Renzulli M, Bolondi L, et al. TACE performed in patients with a single nodule of hepatocellular carcinoma. BMC Cancer. 2014;14:601.

10 Kudo M, Han KH, Ye SL, Zhou J, Huang YH, Lin SM, et al. A changing paradigm for the treatment of intermediate-stage hepatocellular carcinoma: Asia-Pacific primary liver cancer expert consensus statements. Liver Cancer. 2020;9(3):245-60.

11 Yamakado K, Miyayama S, Hirota S, Mizunuma K, Nakamura K, Inaba Y, et al. Hepatic arterial embolization for unresectable hepatocellular carcinomas: do technical factors affect prognosis? Jpn J Radiol. 2012;30(7):560-6.
12 Cammà C, Schepis F, Orlando A, Albanese M, Shahied L, Trevisani F, et al. Transarterial chemoembolization for unresectable hepatocellular carcinoma: meta-analysis of randomized controlled trials. Radiology. 2002;224(1): 47-54.

13 Yip WM, Hung HG, Lok KH, Li KF, Li KK, Szeto ML. Outcome of inoperable hepatocellular carcinoma patients receiving transarterial chemoembolisation: a real-life retrospective analysis in a Hong Kong regional hospital. Hong Kong Med J. 2009;15(5):339-45.

14 Kothary N, Weintraub JL, Susman J, Rundback JH. Transarterial chemoembolization for primary hepatocellular carcinoma in patients at high risk. J Vasc Interv Radiol. 2007; 18(12):1517-27; quiz 27.

15 Golfieri R, Cappelli A, Cucchetti A, Piscaglia F, Carpenzano M, Peri E, et al. Efficacy of selective transarterial chemoembolization in inducing tumor necrosis in small $(<5 \mathrm{~cm})$ hepatocellular carcinomas. Hepatology. 2011; 53(5):1580-9.

16 Kim BK, Kim SU, Kim KA, Chung YE, Kim MJ, Park MS, et al. Complete response at first chemoembolization is still the most robust predictor for favorable outcome in hepatocellular carcinoma. J Hepatol. 2015;62(6):1304-10. 
17 Golfieri R, Renzulli M, Mosconi C, Forlani L, Giampalma E, Piscaglia F, et al. Hepatocellular carcinoma responding to superselective transarterial chemoembolization: an issue of nodule dimension? J Vasc Interv Radiol. 2013;24(4):509-17.

18 Peck-Radosavljevic M, Kudo M, Raoul J-L, Lee HC, Decaens T, Heo J, et al. Outcomes of patients (pts) with hepatocellular carcinoma (HCC) treated with transarterial chemoembolization (TACE): global OPTIMIS final analysis. Jco. 2018;36(15 Suppl):4018.

19 Kudo M, Arizumi T. Transarterial chemoembolization in combination with a molecular targeted agent: lessons learned from negative trials (Post-TACE, BRISK-TA, SPACE, ORIENTAL, and TACE-2). Oncology. 2017; 93(Suppl 1):127-34.

20 Irie T, Takahashi N. Improved accumulation of Lipiodol under balloon-occluded transarterial chemoembolization (B- TACE) for hepatocellular carcinoma: measurement of blood pressure at the embolized artery before and after balloon inflation. Jpn J Intervent Radiol. 2011;26:49-54. (in Japanese).

21 Irie T, Kuramochi M, Takahashi N. Dense accumulation of Lipiodol emulsion in hepatocellular carcinoma nodule during selective balloon-occluded transarterial chemoembolization: measurement of balloon-occluded arterial stump pressure. Cardiovasc Intervent Radiol. 2013;36(3):706-13.

22 Arai $\mathrm{H}$, Abe T, Takayama $\mathrm{H}$, Toyoda M, Ueno T, Kakizaki S, et al. Safety and efficacy of balloon-occluded transcatheter arterial chemoembolization using miriplatin for hepatocellular carcinoma. Hepatol Res. 2015;45(6): 663-6.

23 Hatanaka T, Arai H, Shibasaki M, Tojima H, Takizawa D, Toyoda M, et al. Factors predicting overall response and overall survival in hepatocellular carcinoma patients undergoing balloon-occluded transcatheter arterial chemoembolization: a retrospective cohort study. Hepatol Res. 2018;48(2):165-75.

24 Konno T. Targeting cancer chemotherapeutic agents by use of Lipiodol contrast medium. Cancer. 1990 Nov 1;66(9):1897-903.

25 de Baere T, Arai Y, Lencioni R, Geschwind JF, Rilling W, Salem R, et al. Treatment of liver tumors with Lipiodol TACE: technical recommendations from experts opinion. Cardiovasc Intervent Radiol. 2016 Mar;39(3): 334-43. Epub 2015 Sep 21.

26 Lencioni R, Llovet JM. Modified RECIST (mRECIST) assessment for hepatocellular carcinoma. Semin Liver Dis. 2010;30(1):5260.

27 Filippiadis DK, Binkert C, Pellerin O, Hoffmann RT, Krajina A, Pereira PL. CIRSE quality assurance document and standards for classification of complications: the CIRSE classification system. Cardiovasc Intervent Radiol. 2017;40(8):1141-6.

28 US Department of Health and Human Services. Common Terminology Criteria for Adverse Events (CTCAE).v.5.0; 2017. CTCAE v5
Quick Reference 8.5x11.pdf. Available from: https: //ctep.cancer.gov/protocoldevelopment/electronicapplications/docs/.

29 Miyayama S, Matsui O, Yamashiro M, Ryu Y, Kaito K, Ozaki K, et al. Ultraselective transcatheter arterial chemoembolization with a 2 -f tip microcatheter for small hepatocellular carcinomas: relationship between local tumor recurrence and visualization of the portal vein with iodized oil. J Vasc Interv Radiol. 2007; 18(3):365-76.

30 Uchida H, Ohishi H, Matsuo N, Nishimine K, Ohue S, Nishimura Y, et al. Transcatheter hepatic segmental arterial embolization using Lipiodol mixed with an anticancer drug and Gelfoam particles for hepatocellular carcinoma. Cardiovasc Intervent Radiol. 1990;13(3): 140-5.

31 Arizumi T, Ueshima K, Chishina H, Kono M, Takita M, Kitai S, et al. Validation of the criteria of transcatheter arterial chemoembolization failure or refractoriness in patients with advanced hepatocellular carcinoma proposed by the LCSGJ. Oncology. 2014;87(Suppl 1):32-6.

32 Raoul JL, Forner A, Bolondi L, Cheung TT, Kloeckner R, de Baere T. Updated use of TACE for hepatocellular carcinoma treatment: How and when to use it based on clinical evidence. Cancer Treat Rev. 2019;72:2836.

33 Irie $\mathrm{T}$, Kuramochi M, Kamoshida T, Takahashi N. Selective balloon-occluded transarterial chemoembolization for patients with one or two hepatocellular carcinoma nodules: Retrospective comparison with conventional super-selective TACE. Hepatol Res. 2016; 46(2):209-14.

34 Maruyama M, Yoshizako T, Nakamura T, Nakamura M, Yoshida R, Kitagaki H. Initial experience with balloon-occluded trans-catheter arterial chemoembolization (B-TACE) for hepatocellular carcinoma. Cardiovasc Intervent Radiol. 2016;39(3):359-66.

35 Minami Y, Minami T, Chishina H, Arizumi T, Takita M, Kitai S, et al. Balloon-occluded transcatheter arterial chemoembolization for hepatocellular carcinoma: a single-center experience. Oncology. 2015;89(Suppl 2):27-32.

36 Lammer J, Malagari K, Vogl T, Pilleul F, Denys A, Watkinson A, et al. Prospective randomized study of doxorubicin-eluting-bead embolization in the treatment of hepatocellular carcinoma: results of the PRECISION V study. Cardiovasc Intervent Radiol. 2010; 33(1):41-52.

37 Facciorusso A, Di Maso M, Muscatiello N. Drug-eluting beads versus conventional chemoembolization for the treatment of unresectable hepatocellular carcinoma: a metaanalysis. Dig Liver Dis. 2016;48(6):571-7.

38 Han T, Yang X, Zhang Y, Li G, Liu L, Chen T, et al. The clinical safety and efficacy of conventional transcatheter arterial chemoembolization and drug-eluting beads-transcatheter arterial chemoembolization for unresectable hepatocellular carcinoma: a meta-analysis.
Biosci Trends. 2019 Nov 13;13(5):374-81. Epub 2019 Oct 14.

39 Lucatelli P, Ginnani Corradini L, De Rubeis G, Rocco B, Basilico F, Cannavale A, et al. Balloon-occluded transcatheter arterial chemoembolization (b-TACE) for hepatocellular carcinoma performed with polyethylene-glycol epirubicin-loaded drug-eluting embolics: safety and preliminary results. Cardiovasc Intervent Radiol. 2019;42(6):853-62.

40 Goldman DT, Singh M, Patel RS, Nowakowski FS, Bishay V, Ranade M, et al. Balloon-occluded transarterial chemoembolization for the treatment of hepatocellular carcinoma: a single-center US preliminary experience. J Vasc Interv Radiol. 2019;30(3):342-6.

41 Bucalau AM, Tancredi I, Pezzullo M, Leveque R, Picchia S, Van Laethem JL, et al. Balloonoccluded chemoembolization for hepatocellular carcinoma: a prospective study of safety, feasibility and outcomes. Hepat Oncol. $14 \mathrm{Oct}$ 2020. Published online.

42 Hatanaka T, Arai H, Kakizaki S. Balloon-occluded transcatheter arterial chemoembolization for hepatocellular carcinoma. World J Hepatol. 2018;10(7):485-95.

43 Kawamura Y, Ikeda K, Fujiyama S, Hosaka T, Kobayashi M, Saitoh S, et al. Usefulness and limitations of balloon-occluded transcatheter arterial chemoembolization using miriplatin for patients with four or fewer hepatocellular carcinoma nodules. Hepatol Res. 2017;47(4):338-46.

44 Asayama Y, Nishie A, Ishigami K, Ushijima Y, Takayama Y, Okamoto D, et al. Hemodynamic changes under balloon occlusion of hepatic artery: predictor of the short-term therapeutic effect of balloon-occluded transcatheter arterial chemoLipiodolization using miriplatin for hepatocellular carcinoma. Springerplus. 2016;5:157.

45 Gaba RC, Schwind RM, Ballet S. Mechanism of action, pharmacokinetics, efficacy, and safety of transarterial therapies using ethiodized oil: preclinical review in liver cancer models. J Vasc Interv Radiol. 2018 Mar; 29(3):413-24.

46 Tsai TJ, Chau GY, Lui WY, Tsay SH, King KL, Loong CC, et al. Clinical significance of microscopic tumor venous invasion in patients with resectable hepatocellular carcinoma. Surgery. 2000;127:603-8.

47 Esnaola NF, Lauwers GY, Mirza NQ, Nagorney DM, Doherty D, Ikai I, et al. Predictors of microvascular invasion in patients with hepatocellular carcinoma who are candidates for orthotopic liver transplantation. J Gastrointest Surg. 2002;6:224-32.

48 Sasaki A, Kai S, Iwashita Y, Hirano S, Ohta M, Kitano S. Microsatellite distribution and indication for locoregional therapy in small hepatocellular carcinoma. Cancer. 2005 Jan 15; 103(2):299-306.

49 Choi JW, Park JY, Ahn SH, Yoon KT, Ko HK, Lee DY, et al. Efficacy and safety of transarterial chemoembolization in recurrent hepatocellular carcinoma after curative surgical resection. Am J Clin Oncol. 2009;32(6):564-9. 
50 Ebied OM, Federle MP, Carr BI, Pealer KM, Li W, Amesur N, et al. Evaluation of responses to chemoembolization in patients with unresectable hepatocellular carcinoma. Cancer. 2003;97(4):1042-50.

51 Takayasu K. Superselective transarterial chemoembolization for hepatocellular carcinoma: recent progression and perspective. Oncology. 2011;81(Suppl 1):105-10.

52 Terayama N, Matsui O, Gabata T, Kobayashi S, Sanada J, Ueda K, et al. Accumulation of iodized oil within the nonneoplastic liver adjacent to hepatocellular carcinoma via the drainage routes of the tumor after transcatheter arterial embolization. Cardiovasc Intervent Radiol. 2001;24(6):383-7.

53 Matsuo N, Uchida H, Nishimine K, Soda S, Oshima M, Nakano H, et al. Segmental transcatheter hepatic artery chemoembolization with iodized oil for hepatocellular carcinoma: antitumor effect and influence on normal tissue. J Vasc Interv Radiol. 1993;4(4):543-9.
54 Matsui O, Kadoya M, Yoshikawa J, Gabata T, Arai K, Demachi H, et al. Small hepatocellular carcinoma: treatment with subsegmental transcatheter arterial embolization. Radiology. 1993;188(1):79-83.

55 Kuroda C, Sakurai M, Monden M, Marukawa $\mathrm{T}$, Hosoki T, Tokunaga K, et al. Limitation of transcatheter arterial chemoembolization using iodized oil for small hepatocellular carcinoma. A study in resected cases. Cancer. 1991; 67(1):81-6.

56 Kojiro M, Sugihara S, Kakizoe S, Nakashima O, Kiyomatsu K. Hepatocellular carcinoma with sarcomatous change: a special reference to the relationship with anticancer therapy. Cancer Chemother Pharmacol. 1989;23 Suppl:S4-8.

57 Wang B, Xu H, Gao ZQ, Ning HF, Sun YQ, Cao GW. Increased expression of vascular endothelial growth factor in hepatocellular carcinoma after transcatheter arterial chemoembolization. Acta Radiol. 2008;49(5):523-9.
58 Zen C, Zen Y, Mitry RR, Corbeil D, Karbanová J, O'Grady J, et al. Mixed phenotype hepatocellular carcinoma after transarterial chemoembolization and liver transplantation. Liver Transpl. 2011;17(8):943-54.

59 Miyayama S, Matsui O, Zen Y, Yamashiro M, Hattori Y, Orito N, et al. Portal blood supply to locally progressed hepatocellular carcinoma after transcatheter arterial chemoembolization: Observation on CT during arterial portography. Hepatol Res. 2011;41(9):853-66.

60 Miyayama S, Yamashiro M, Sugimori N, Ikeda R, Okimura K, Sakuragawa N. Outcomes of patients with hepatocellular carcinoma treated with conventional transarterial chemoembolization using guidance software. J Vasc Interv Radiol. 2019 Jan;30(1):10-8.

61 Ishikawa $\mathrm{T}, \mathrm{Abe} \mathrm{S}$, Inoue R, Sugano T, Watanabe $\mathrm{Y}$, Iwanaga A, et al. Predictive factor of local recurrence after balloon-occluded TACE with miriplatin (MPT) in hepatocellular carcinoma. PLoS One. 2014;9(7):e103009. 